\title{
Enterprise-wide versus Best of Breed manufacturing solutions: providing a structured approach to systems strategy selection
}

\author{
C.J. Gavin and P. Cheshire
}

Sema Group

Lindsay House

167/169 Shaftesbury Avenue

London WC2H $8 A N$

Tel: +441718304444

Fax: +441713790669

Email:Carl.Gavin@mail.sema.co.uk,

Paul.Cheshire@mail.sema.co.uk

\begin{abstract}
Many manufacturing organisations embarking upon new systems implementation projects face a choice between an integrated ERP package or separate Best Of Breed components. Drawing from work in this area, this paper presents the cases for and against the options met, outlining the key technical, financial and human factors which have to be considered. Based upon the need for a structured approach in understanding and resolving the associated issues, key decision criteria facing manufacturing companies in the choice of an appropriate system are presented.
\end{abstract}

\section{Keywords}

Manufacturing information systems, Enterprise Resource Planning (ERP), best of breed, packaged systems 


\section{INTRODUCTION}

When embarking upon new systems implementation projects in support of the manufacturing value chain, companies face a fundamental decision: to focus only on the critical systems components which are "life expired" or which simply do not exist, or to replace all or the bulk of their existing application portfolio. The business benefits for either approach have to be carefully evaluated - it may be cost-effective to consider widening the scope of the project beyond its core focus. If there are to be quantifiable benefits from taking a wide-ranging approach, three main options exist:

- to buy a wholly or mostly bespoke solution, presumably from one vendor.

- to buy one integrated "enterprise-wide" or Enterprise Resource Planning package from one vendor (for example, SAP or Baan).

- to buy a range of "best of breed" packages from a number of vendors.

\section{Bespoke solutions}

In the manufacturing systems marketplace, commissioning bespoke solutions is no longer seriously considered. Even so, a major revenue stream for software vendors comes from tailoring packaged solutions. The practical choice for a manufacturing organisation comes down to the preference for the "enterprise-wide" solution or the adoption of "best of breed" packages. Both are concerned with choosing and integrating core applications.

\section{Enterprise Resource Planning (ERP)}

The growth in the ERP market has been staggering and is predicted to continue, with large numbers of medium to large manufacturing organisations yet to invest in such solutions. The ideal solution promised by ERP vendors is the replacement of the disparate systems supporting finance, manufacturing, distribution, sales, and so on, with just one application, one user interface and one database. According to a recent evaluation of ERP packages (Evans et al, 1997), this promise remains undelivered: many of the functions and applications provided are only loosely integrated. Initial ERP solutions have extended MRPII and focused upon the core applications within manufacturing companies: manufacturing, distribution planning and corporate accounting systems. A key problem is that in reality, many manufacturing companies are unsure of what ERP can provide. It has often been stated that ERP seems to be "all things to all men".

ERP as a concept is of a wholly integrated set of business applications that assists everyone in an enterprise, employees and managers alike, to plan, monitor and control their part of the business (Waldron, 1992; Ricciuti, 1992; Hicks and Stecke, 1995; Parker, 1996). It affords them degrees of freedom and authority within their domain of control, but it imposes constraints and processes to ensure that the business operates in accordance with well defined principles, goals and objectives. The concept demands a computer system capable of excellence in each 
given functional area and with degrees of integration as high as those in a business whose management and operational activities are conducted in a fashion that is "better than best practice".

The business drivers for embracing the ERP concept include on-line financial control; enhancing a company's ability to schedule production accurately; making best use of operational capacity; reducing inventory; and being able to guarantee and to meet shipping dates.

A typical ERP package goes some way towards fulfilling the vision and promise of "best practice". Some packages are more advanced than others in terms of:

- the scope of business and operational functions which are supported.

- the degree to which those individual functions match the way of working that an organisation has adopted.

- the degree to which the functions actually integrate.

\section{Scope}

In terms of scope, most packages offer sales and purchase planning; distribution planning; manufacturing planning; and corporate accounting. Some go further, and provide support for human resources; corporate management and strategy; sales and marketing automation; new product and process development and manufacturing process automation.

It is important to recognise that the scope of functionality available may be greater than a business actually intends to use. Sometimes the "extras" are beneficial; sometimes they are irrelevant to the organisation. There is, however, no simple universal rule which can help decide if a given "feature" is a benefit or a millstone.

\section{Supporting a way of working}

The "fit" between the way a package works and the way a business works also varies. One school of thought suggests that the best approach is to adopt a welldefined set of business processes and the implied organisational structure as directly supported by the package; others use the flexibility of the package to support a variety of business operational paradigms and they configure a package to match their way of working.

This "fit" also needs to be considered in terms of the actual operational methods needed, since these will be quite different in different types of organisation (for example, the petrochemical industries work in a way which is markedly different from aircraft construction). The way in which key performance indicators are delivered to managers also needs to be taken into account. In addition, thought needs to be given to how managers will use the information from the system to optimise activities which are failing to meet targets or which are capable of achieving higher targets than those which have been set. 


\section{Delivering a single consistent view of the enterprise}

Finally, we can consider the way in which the components integrate. Some take information from every function and store this in a single database accessible by all other functions; others pass messages between functions, leaving the issue of data storage and ensuring consistency to each separate function. The timeliness and sophistication of such messaging varies significantly from "immediate" transfers of each relevant item to "overnight batch file transfers". Neither approach is inherently wrong.

\section{The cost}

A "full" ERP system is a significant investment for any company, perhaps taking up to $3 \%$ of turnover or $30 \%$ of IT budget during its implementation and "settling in" period. ERP is not immune from the troubles that often beset large systems implementations: deadlines are not met; partial functionality is delivered; costs escalate; and end user expectations exceed the capabilities of the system. Avoiding such risks in an ERP implementation requires the same skills as in any other systems-related project, for example, a focus on the core needs; commitment from the key stakeholders; management of expectations; and training. The key objective is to manage costs and draw through the benefits of use the system as rapidly as possible.

In practice, every implementation is unique, with the mixing and matching of functions available from a standard product, with bespoke developments, tailoring and customisation where appropriate. It is rare to find an ERP implementation which can start with a completely clean sheet of paper: there will be legacy systems which must be preserved; and there will be information locked away in application repositories which has to find its way into the new application. In poorly planned projects, the full cost and implications of dealing with legacy systems often emerge too late.

\section{Best Of Breed}

A best of breed approach to providing key functions would appear to be a more realistic option, and one based upon more traditional approaches. The promise of this approach is the realisation of the benefits of integrated systems without the need, or indeed the opportunity, to purchase all the applications from a single software vendor, either at the outset or subsequently when a "better" application becomes available. Such an approach will be essential if the ERP vision remains unfulfilled. However, this choice is also not without problems.

The main problem with the best of breed approach is the difficulty in achieving a satisfactory level of systems integration between the individual components. The need to integrate legacy applications with the new systems remains, but the costs and implications are potentially amplified because the issues are both immediately obvious and involve many suppliers. 
Integration is often more of an afterthought than a strategic direction, the pressing problem being the lack of standards and interoperability. One advantage of the ERP over Best of Breed is that there is a (proprietary) standard for integrating applications within the ERP application domain.

\section{The most appropriate solution}

The key issue is the definition of the most appropriate solution, the one that best meets the core needs. The days are of bespoke development are past; the packages are mature and have evolved to meet the needs of industry as it too has evolved. The decision of whether to invest in best of breed or single source needs to be tackled by breaking down the question into more manageable parts.

We can make comparisons of the cost of purchase - often this is a critical criterion because all organisations need to manage their cash flow. Additionally we can look at total cost of ownership and make judgements based on the anticipated whole lifetime costs, and not just the one-off implementation costs. Such an approach is more common in "forward looking" organisations where consideration is given to how the system is predicted to change over time in response to changes in business needs as well as technology-led changes. We can also assess how the use of the system can remove costs from the business, for example electronic document exchanges may be cheaper and more reliable than paper transfers.

We can make objective comparisons of the packages against the business requirements and assess which approach delivers the best functional fit. In making such an evaluation it's important to decide if the package or established practice will be the yardstick for the way of working. There is usually a sensible balance to be struck and it is entirely possible that the new solution will offer the buyer the opportunity to learn more about, and exploit, trends in industry practices.

We can also give consideration to the value of the system and assess how much value may be added to the enterprise (the philosophy of adding value is quite different from removing costs from the business). For example, we may aim to provide better customer service and thus attract a greater market share without making any change to nature or quality of our product.

\section{PROVIDING A STRUCTURED APPROACH}

Manufacturing planning and control is now a complex area, with a considerable number of approaches and packages to choose from. Indeed, the level of choice can be daunting. Previous research has identified the problems inherent in the specification and selection of an appropriate information system and of the need for a structured approach to ease such problems (Gavin and Little, 1994; Gavin and Little, 1995; Kochhar et al, 1995; Little, Gavin et al, 1997; Maull and Childe, 1993; Muhlemann et al, 1995). The main issues cited include poor specification of user needs, politics, a lack of formal methods, and the selection of inappropriate systems. The issue of ERP versus Best of Breed solutions outlined in this paper 
intensifies the issues of selection. It is important that the structured approach employed can support and balance the key business needs. The authors advocate an approach based upon the balanced business scorecard, a performance measurement framework that can help translate the strategic objectives of a company into specific goals and provide a focus to the many changes that may occur.

\section{BALANCING THE NEEDS OF THE BUSINESS}

There are four crucial performance areas which any business needs to manage, namely:

- money

- operational efficiency and effectiveness

- innovation and learning

- customer service.

Ultimately, all decision factors may be grouped into these four headings and each may be quantified to permit objective comparison.

For an ERP implementation, there are expected costs and benefits. For example, a benchmark investment cost is $3 \%$ of turnover. Operational efficiency should be improved in most departments. Reductions of $30-60 \%$ in ERP users' long-term information systems budgets are typical since the cost of maintaining disparate and poorly integrated legacy systems is replaced by the cost of maintaining a system designed with integration in mind. Most administrative departments can complete their tasks more rapidly and using fewer staff. Improved operational control, of inventory, work-in-progress and manufacturing resources releases money into the business. Integrating the manufacturing and distribution processes and information results in plans which are challenging yet realistic and achievable.

Investment in ERP systems improves a company's effectiveness. Employees at all levels in the company are encouraged to follow well defined processes and procedures which have been designed with repeatability, effectiveness and manageability in mind. Information that is needed to manage individual processes and the business is accessible and available to help managers to make better decisions more rapidly. Integration with financial data supports activity-based and other costing models.

The cost of purchase may be higher for a multi-sourced solution. The cost (and risk) of integrating multi-source components will be higher than buying a "ready integrated" package. In the multi-source scenario there will be more gaps to find and to fill and potentially more overlaps to resolve. There is a lack of consistent use of standard connectivity tools. The cost of maintaining software and any bespoke interfacing and integration code is likely to be lower for a single source solution.

Niche vendors, however, often have products which are leaders in their particular fields. These products may offer "the best" implementation of a given function; 
and it may be easier to improve a businesses overall performance by having a collection of small modules, each of which may be optimised within an overall corporate scheme.

Single-source vendors are making improvements in their use of integration and interfacing technologies which will allow true Best of Breed components to be integrated. Best of Breed vendors are doing the same to advance their integration capability whilst still striving to advance their "niche" capabilities faster than the single source vendors. The natural consequence of this is that the best of breed and the single source vendors will collaborate such that the single source vendor offers the commercial and technical framework, "the commodity" software and the "integration services" to build the required solution from the most appropriate sources.

Industry standard business process and operational models are being used by many vendors to provide better delineation of functions in order to reduce gaps and overlaps which in turn will reduce the complexity of interaction and integration. In addition, more "build time" configuration options are being provided. This means that smaller modules appropriate for a specific niche can be included in a systems, rather than including a module which may, by inspecting "configuration switches", determine its run-time characteristics. For example, a system may have different three different "payroll" modules available; each designed for a specific type of need rather than one "utility" payroll which needs to be extensively configured to permit the right options to be invoked.

\section{SUMMARY}

Growth in the market for enterprise-wide systems has been impressive, but only a few vendors have a full suite of ERP modules available and far from all potential customers have implemented the whole range. The next few years will see the larger manufacturers investing in additional modules; and the medium-sized enterprises, currently shunning large and expensive systems, will be attracted by falling one-off costs. This means user demand, rather than technology change, will drive product development. The need for a structured approach remains, to help manufacturing systems engineers and users define the most appropriate solution. The authors suggest an approach based upon balancing four critical performance areas.

\section{REFERENCES}

Evans, M., Bragg, S. and Kleevers, T. (1997). Ovum evaluates: ERP for manufacturers. Ovum, UK.

Gavin, C.J. and Little, D. (1994). The application of case within manufacturing industry. Software Engineering Journal - Special Issue on CASE, 9(4), 140152. 
Gavin, C.J. and Little, D. (1995). Structured approaches to the definition of manufacturing planning and control systems. Proceedings of the IFIP WG5.7 Conference on Concurrent Manufacturing, Seattle, USA.

Hicks, D.A: and Stecke, K.E. (1995). The ERP maze. IIE Solutions, 27(8), 12-16.

Kochhar, A.K., Oldham, K. And Thacker, S.M. (1995). Structured methodology for the selection and effective implementation of manufacturing control systems. IEE Proceedings - Sci. Meas. Technol., 142(5), 411-416.

Little, D., Gavin, C.J., Guenaoui, D. And Hodgson, N. (1997). Survey of SME information systems planning and operation. Control, 23(5), 17-20.

Maull, R.S. and Childe, S.J. (1993). A step-by-step guide to the identification of an appropriate computer-aided production management system. Production Planning and Control, 4(1), 69-76.

Muhlemann, A., Price, D., Afferson, M. And Sharp, J. (1995). A computer based approach for enhancing manufacturing decision making in smaller manufacturing enterprises: a longitudinal study. International Journal of Management Science, 23(1), 97-107.

Parker, K. (1996). The Enterprise endeavour. Manufacturing Systems, January 1996, 14-30.

Ricciuti, M. (1992). Connect manufacturing to the enterprise. Datamation, 38(2), 42-44.

Waldron, D. (1992). What follows MRPII? Enterprise resource planning. Professional Engineering, May 1992, 22-23.

\section{BIOGRAPHIES}

Mr Paul Cheshire is a Consulting Director in Sema Group and head of the company's Manufacturing Centre of Excellence. He has spent 25 years developing and implementing computer-based systems to meet business and industrial requirements. His first-hand experience of building and presenting the rationale for particular systems strategies comes from active involvement in project work across a range of industries from board-room to shop floor and from his participation in national and international manufacturing technology standards.

Before recently joining Sema Group as Managing Consultant, Dr Carl Gavin was Director of Studies and Lecturer in Manufacturing Management in the Department of Manufacturing Engineering and Industrial Management at the University of Liverpool. Carl's doctoral research, sponsored by Andersen Consulting, investigated structured approaches to manufacturing information systems development and this formed the basis for recent research projects. Carl is currently a member of the National Council of the Institute of Operations Management, an Honorary Lecturer at the University of Liverpool and a Visiting Research Fellow at the University of Huddersfield. 